May 19, 2018

WM-97-115

\title{
Supersymmetric model of quasi-degenerate neutrinos
}

\author{
Christopher D. Carone and Marc Sher \\ Nuclear and Particle Theory Group \\ Department of Physics \\ College of William and Mary \\ Williamsburg, VA 23187-8795
}

\begin{abstract}
We present a supersymmetric model of fermion masses, based on a nonAbelian family symmetry and the Froggatt-Nielsen mechanism, that can account for the solar and atmospheric neutrino problems via quasi-degenerate neutrinos. The model predicts that the ratio of neutrino mass squared splittings $\Delta m_{12}^{2} / \Delta m_{23}^{2}$ is of order $m_{s}^{2} / m_{b}^{2}$, and the angles $\theta_{12} \sim m_{d} / m_{s}$ and $\theta_{23} \sim 1$, which are of the desired orders of magnitude. We discuss the implications of the flavor structure of the neutrino sector on superparticle masses and mixing angles.
\end{abstract}

*This work was supported in part by the National Science Foundation under grant PHY-96-00415. 


\section{Introduction}

The most striking feature of the quark and charged lepton masses is their hierarchical structure. An entire industry has developed in an attempt to understand this pattern, most recently in the context of supersymmetric theories [1]. In this letter we will construct a supersymmetric model of flavor with the physics of the neutrino sector specifically in mind.

Given the hierarchy of the charged fermion masses, it is natural to assume that the neutrino masses follow a similar hierarchical structure: $m_{\nu_{e}}<<m_{\nu_{\mu}}<<m_{\nu_{\tau}}$. However, we will consider an alternative possibility, that the three generations of neutrinos are nearly degenerate, with small splittings sufficient to explain (i) the solar neutrino problem via $\nu_{e}-\nu_{\mu}$ MSW oscillations, and (ii) the atmospheric neutrino problem via $\nu_{\mu}-\nu_{\tau}$ mixing [2]. While these effects can be explained in models with hierarchical neutrino masses as well, it is important to explore all the possibilities, especially in light of the evidence from the SuperKamiokande experiment that neither the solar nor atmospheric neutrino problems are about to disappear [3]. A number of interesting theories with quasi-degenerate neutrinos can be found in the recent literature 4 4, 6, 7], and given the improvement in the experimental situation, it is worthwhile to give this and other scenarios further consideration.

Our model is based on a global non-Abelian family symmetry that restricts the quark, lepton, neutrino, and scalar superparticle mass matrices simultaneously; we don't consider the lepton sector in isolation, unlike some of the other models on the market. We assume flavor-symmetry breaking originates in the fermion Yukawa couplings via a sequential breaking of the flavor symmetry groupt. Hence, we avoid any ansatz for the Yukawa textures that cannot be motivated from symmetry considerations. Interestingly, the elements of the Yukawa matrices in our model that control right-handed rotations in the quark sector largely determine the neutrino mixing angles. This provides another constraint on the form of the Yukawa textures. We show that the model can solve problems (i) and (ii) above via the seesaw mechanism, yielding the appropriate neutrino mass ratios and mixing angles in terms of ratios of quark Yukawa couplings. In addition, the model solves the supersymmetric flavor problem by yielding superparticle degeneracies in the flavor symmetric limit. With the origin of flavor symmetry breaking specified, we consider the detailed form of the superparticle

\footnotetext{
${ }^{\dagger}$ The symmetry-breaking scale is taken high enough so that the associated goldstone boson decay constants are above the lower bounds from direct collider searches for familons.
} 
mass matrices and state the distinctive supersymmetric signatures of the model.

\section{The Model}

In the limit of vanishing Yukawa couplings, the supersymmetric standard model has a global $U(3)^{5}$ flavor symmetry, with a separate $\mathrm{U}(3)$ factor acting on each of the chiral superfields $Q, U, D, L$, and $E$. One approach to constructing models of flavor is to impose a horizontal symmetry that is a subgroup of $\mathrm{U}(3)_{Q} \times \mathrm{U}(3)_{U} \times \mathrm{U}(3)_{D}$ alone, where $\mathrm{U}(3)_{Q}$ and $\mathrm{U}(3)_{D}$ are assumed to act on the lepton fields $L$ and $E$. In this way, the known similarity between the down quark and charged lepton masses [8] can be understood, up to factors of order unity. This approach was taken in Refs. [9, 10] and will also be adopted here. While this choice leaves a twofold ambiguity in assigning the $L$ and $E$ transformation properties, only one assignment, in which $L$ and $E$ transform under $\mathrm{U}(3)_{D}$ and $\mathrm{U}(3)_{Q}$, respectively, will lead to the desired pattern of neutrino masses and mixing angles. We must first decide on a subgroup of $\mathrm{U}(3)^{3}$ to use in constructing a model, and specify the transformation properties of the right-handed neutrino fields, $\nu$.

Since our goal is to construct a model of quasi-degenerate neutrino masses, we will require the mass matrix of the light neutrino mass eigenstates to be proportional to the identity in the flavor-symmetric limit. Since this mass matrix arises via the see-saw mechanism,

$$
M_{L L} \approx M_{L R} M_{R R}^{-1} M_{L R}^{T}
$$

we can achieve degeneracy if the Dirac and Majorana mass matrices, $M_{L R}$ and $M_{R R}$, are individually proportional to the identity. If the lepton doublet $L$ is in a three dimensional representation of some subgroup of $\mathrm{U}(3)_{D}$, then we can achieve the desired form of $M_{L R}$ if $\nu$ transforms in the $\overline{\mathbf{3}}$ (assuming the Higgs fields are singlets). However, we also require $M_{R R}$ to be invariant under the flavor symmetry and proportional to the identity, which implies that $\overline{\mathbf{3}} \sim \mathbf{3}$; thus we seek a subgroup of $\mathrm{U}(3)_{D}$ that has real three-dimensional representations. The largest subgroup that is appropriate is $\mathrm{SO}(3)$, which we will adopt henceforth. As for the part of the flavor symmetry that lives in the factors $\mathrm{U}(3)_{Q} \times \mathrm{U}(3)_{U}$, we will take the largest symmetry possible, $\mathrm{U}(3)_{Q} \times \mathrm{U}(3)_{U}$ itself. Thus, we begin with the maximal flavor symmetry group appropriate for our purposes,

$$
G_{f}=U(3)_{Q} \times U(3)_{U} \times S O(3)_{D}
$$


where the three generations of left- and right-handed neutrinos transform as three dimensional vectors under $\mathrm{SO}(3)_{D}$.

The top quark Yukawa coupling breaks $G_{f}$ strongly down to

$$
G_{f}^{e f f}=U(2)_{Q} \times U(2)_{U} \times U(1)_{Q_{3}-U_{3}} \times S O(3)_{D}
$$

where the $\mathrm{U}(1)$ factor rotates $Q_{3}$ and $U_{3}$ by an opposite phase. This is the approximate flavor symmetry relevant at low energies, with the light fermion Yukawa couplings treated as small symmetry breaking parameters. Notice that the large top quark Yukawa coupling does not break the $\mathrm{SO}(3)$ symmetry, so we don't expect any large deviation from degeneracy in the neutrino sector. To properly construct the low-energy effective theory, we consider all operators invariant under $G_{f}^{e f f}$, with the light fermion Yukawa couplings included as small symmetry-breaking parameters. Alternatively, we could directly impose $G_{f}^{e f f}$ as the high-energy flavor symmetry, in which case an order one top quark Yukawa coupling would follow as a prediction of the theory.

If we assume that the light fermion Yukawa couplings are the only source of flavor symmetry breaking, we can estimate the symmetry breaking effect in any operator of interest in the low-energy effective theory [12]. Suppose that the Yukawa couplings in the quark sector originate from fields that transform simultaneously under pairs of the group factors in Eq. (2.2)

$$
\Phi_{u} \sim(\overline{\mathbf{3}}, \overline{\mathbf{3}}, \mathbf{1}) \quad, \Phi_{d} \sim(\overline{\mathbf{3}}, \mathbf{1}, \mathbf{3})
$$

Here we state $G_{f}$ transformation properties of the fields for notational convenience only. The reader should keep in mind that this is simply a shorthand for representing the set of fields contained in the $G_{f}^{e f f}$ decomposition of the $\Phi$. Using these fields, we may write down the following higher-dimensional superpotential interactions

$$
W=\frac{1}{M_{F}}\left(Q \Phi_{u} H_{u} U+Q \Phi_{d} H_{d} D\right)
$$

where $M_{F}$ is the flavor scale. We will specify the origin of these operators below. Yukawa couplings arise if the $\Phi$ acquire an appropriate pattern of vacuum expectation values (vevs). For example, let us consider the following pattern of vevs, given in units of $M_{F}$ :

$$
\Phi_{d}=\left(\begin{array}{ccc}
h_{d} & h_{s} \lambda & h_{b} V_{u b} \\
h_{d} & h_{s} & h_{b} V_{c b} \\
h_{d} & h_{b} & h_{b}
\end{array}\right)
$$




\begin{tabular}{cc} 
& $\mathrm{U}(3)_{Q} \times \mathrm{U}(3)_{U} \times \mathrm{SO}(3)_{D}$ \\
\hline \hline$h_{t}$ & $\mathrm{U}(2)_{Q} \times \mathrm{U}(2)_{U} \times \mathrm{U}(1)_{Q_{3}-U_{3}} \times \mathrm{SO}(3)_{D}$ \\
$h_{b}$ & $\mathrm{U}(2)_{Q} \times \mathrm{U}(2)_{U} \times Z_{2}$ \\
$h_{c}$ & $\mathrm{U}(1)_{Q_{1}} \times \mathrm{U}(1)_{U_{1}} \times \mathrm{U}(1)_{Q_{2}-U_{2}} \times Z_{2}$ \\
$h_{s}, h_{b} V_{c b}$ & $\mathrm{U}(1)_{Q_{1}} \times \mathrm{U}(1)_{U_{1}} \times Z_{2}$ \\
$h_{s} \lambda, h_{b} V_{u b}$ & $\mathrm{U}(1)_{U_{1}} \times Z_{2}$ \\
$h_{d}$ & $\mathrm{U}(1)_{U_{1}}$ \\
$h_{u}$ & nothing \\
\hline
\end{tabular}

Table 1: Sequential symmetry breaking leading to the mass matrices in Eqs. (2.6) and (2.7) The $\mathrm{U}(1)_{X_{i}}$ act on the $X$ superfield of the $i^{\text {th }}$ generation, and the $Z_{2}$ flips the sign of the superfield $D_{1}$.

$$
\Phi_{u}=\left(\begin{array}{ccc}
h_{u} & h_{s} \lambda & h_{s} \lambda \\
h_{u} & h_{c} & h_{s} \\
h_{u} & h_{s} & h_{t}
\end{array}\right) .
$$

Here, the $h_{i}$ are the quark Yukawa couplings, $\lambda \approx 0.22$ is the Cabibbo angle, and the $V_{i j}$ are Cabibbo-Kobayashi-Maskawa (CKM) mixing angles. Order one coefficients have been suppressed. The diagonal elements yield the quark mass eigenvalues, while the entries that determine rotations on the left-handed down quark fields reproduce the CKM angles [11. It is interesting that the remaining elements, which control righthanded rotations in the quark sector, will give us the desired flavor structure in the neutrino sector of the theory. An important point is that the hierarchy of entries in Eqs. (2.6) and (2.7) can be understood in terms of a sequential breaking of the original symmetry group $G_{f}$, through a series of subgroups at successively lower scales below $M_{F}$. We may assume that each of these scales is associated with one or more $\Phi$ fields that acquire the most general set of vevs consistent with with the unbroken flavor symmetries at that scale; elements of the Yukawa matrices that differ hierarchically originate from the vevs of different $\Phi$ fields. The textures in Eqs. (2.6) and (2.7) then arise naturally given the symmetry breaking pattern shown in Table. 1.

We may now treat the Yukawa couplings in Eqs. (2.6) and (2.7) as small symmetry breaking parameters, and estimate the size of $G_{f}^{e f f}$-violation in any $1 / M_{F}$ suppressed operators of interest in the low-energy effective theory. This will allow us to determine the sizes of squark, slepton, and neutrino nondegeneracies. The nonrenormalizable 

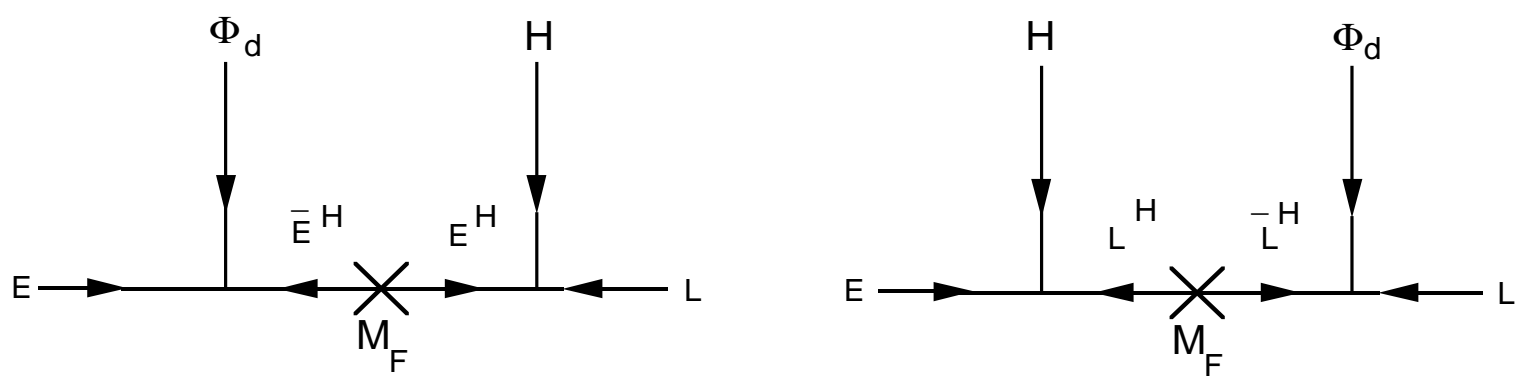

Figure 1: Superfield diagrams contributing to the charged lepton Yukawa matrix.

operators that we study may arise in a theory that is renormalizable at high energies if we work below the scale at which heavy states have been integrated out, as in the approach of Froggatt and Nielsen [13]. In our model, we may implement this mechanism by introducing three 15-plet generations of vector-like fields at the scale $M_{F}$ that we integrate out at lower scales. Denoting these heavy fields with the superscript $H$, the desired interactions are obtained providing that $D^{H}, U^{H}$, and $L^{H}$ transform as fundamentals under $\mathrm{U}(3)_{Q}$, while $E^{H}$ and $Q^{H}$ transform under $\mathrm{SO}(3)_{D}$. For example, for the leptons, we have superpotential interactions of the form

$$
W=\bar{L}^{H} \Phi_{d} L+L^{H} H_{d} E+\bar{E}^{H} \Phi_{d} E+E^{H} H_{d} L+M_{F}\left(\bar{E}^{H} E^{H}+\bar{L}^{H} L^{H}\right),
$$

which produce the charged lepton Yukawa matrix via the two superfield diagrams shown in Figure 1. The fact that the Yukawa interactions arise via renormalizable interactions will affect the form of the neutrino mass matrices, as we shall see shortly.

We now consider the phenomenology of the neutrino sector of the theory. The neutrino superpotential is given by

$$
W=\rho \nu \nu+L H_{u} \nu
$$

where $\rho$ is some field whose (B-L breaking) vev determines the right-handed neutrino mass scale. We have explicitly constructed our model so that these interactions are proportional to the identity in flavor space, at lowest order. To find a correction to this result, we must be able to construct a symmetry-breaking operator out of the $\Phi$ that transforms nontrivially under $\mathrm{SO}(3)_{D}$ alone, and is in the product $\mathbf{3} \times \mathbf{3}$. Because of the transformation properties of $\Phi_{d}$ under $\mathrm{U}(3)_{Q}$, the only way we can form a $\mathrm{U}(3)_{Q}$ singlet is through the product $\Phi_{d}^{\dagger} \Phi_{d}$. However, while an operator of the form

$$
\frac{1}{M_{F}^{2}} L H_{u} \Phi_{d}^{\dagger} \Phi_{d} \nu
$$




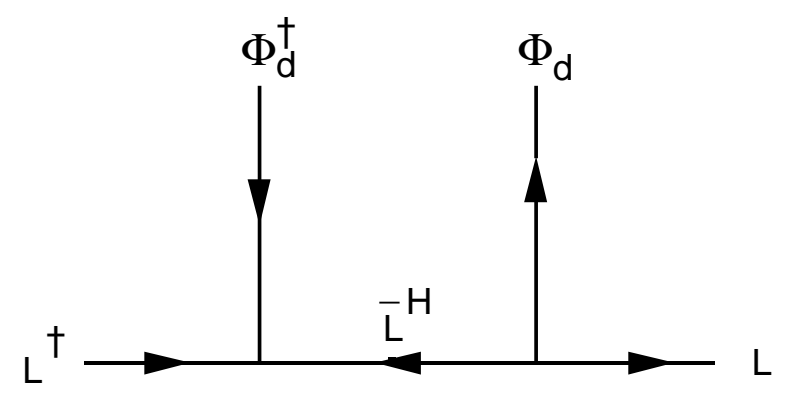

Figure 2: Superfield diagram contributing to Eq. (2.11).

is invariant under the flavor symmetry, it is not a holomorphic function of the fields, and cannot be included in the superpotential. Thus, there is no way we can directly modify Eq. (2.9) by higher-dimensional, $\Phi$-dependent terms in a way that is consistent both with $G_{f}^{e f f}$ invariance, and unbroken supersymmetry.

However, there is an another way in which the exact neutrino degeneracy in Eq. (2.9) may be perturbed, namely through corrections to the Kähler potential of the theory, which is not a holomorphic function of the fields. Let us represent the $\mathrm{U}(2)_{Q}$ singlet and doublet parts of $\Phi_{d}$ as $\Phi_{d}^{(1)}$ and $\Phi_{d}^{(2)}$, respectively. Corrections to the minimal Kähler potential for the left-handed neutrinos of the form

$$
K=L^{\dagger} L+\frac{1}{M_{F}^{2}} L^{\dagger} \Phi_{d}^{(1) \dagger} \Phi_{d}^{(1)} L+\frac{1}{M_{F}^{2}} L^{\dagger} \Phi_{d}^{(2) \dagger} \Phi_{d}^{(2)} L,
$$

force a field redefinition to place the neutrino kinetic terms in canonical form. The correction terms in Eq. (2.11) may arise in our Froggatt-Nielsen model via the superfield diagram in Figure 2. If we write the lowest order field redefinition as $L \rightarrow(1-\Delta / 2) L$, with the matrix $\Delta$ determined from Eq. (2.11), then the original neutrino mass matrix $M_{L L}$, which is proportional to the identity, becomes $M_{L L}\left[1-\left(\Delta-\Delta^{T}\right) / 2\right]$. Using the $\{\Phi\}$ vevs given in Eq. (2.6), we may now construct the neutrino mass matrix. We find

$$
\begin{aligned}
& M_{E} \sim \Phi_{d}^{(1) \dagger}+\Phi_{d}^{(2) \dagger} \approx\left(\begin{array}{ccc}
h_{d} & h_{d} & h_{d} \\
h_{s} \lambda & h_{s} & h_{b} \\
h_{b} V_{u b} & h_{b} V_{c b} & h_{b}
\end{array}\right) \\
& M_{L L} \sim 1+\Phi_{d}^{(1) \dagger^{\prime}} \Phi_{d}{ }^{(1)^{\prime}}+\Phi_{d}^{(2) \dagger^{\prime}} \Phi_{d}^{(2)^{\prime}} \\
& \approx 1+\left(\begin{array}{ccc}
h_{d}^{2} & a h_{d} h_{b}+h_{d} h_{s} & b h_{d} h_{b}+h_{d} h_{b} V_{c b} \\
a h_{d} h_{b}+h_{d} h_{s} & a h_{b}^{2}+h_{s}^{2} & a b h_{b}^{2} \\
b h_{d} h_{b}+h_{d} h_{b} V_{c b} & a b h_{b}^{2} & b^{2} h_{b}^{2}
\end{array}\right) .
\end{aligned}
$$


Here we have included the order one coefficients $a$ and $b$ where they are relevant to our results. We consider these matrices in detail below.

\section{Neutrino Phenomenology}

Neutrino mass splittings. Clearly the neutrino mass splittings are controlled by the correction to the identity matrix shown in Eq. (2.13). Notice that the leading, $\mathcal{O}\left(h_{b}\right)$, correction arises from the coupling of a single linear combination of the $\Phi_{d}^{(1)}$ fields, $\Phi_{d}^{(1)^{\prime}} \sim\left(h_{d}, h_{b}, h_{b}\right)$, that participates in the $\bar{L}^{H} \Phi L$ vertex in Figure 2. As a consequence, the 2-3 block in Eq. (2.13) must have one vanishing eigenvalue, which is corrected at order $h_{s}^{2}$. The extent to which this eigenvalue relation can be preserved will be discussed in the section on fine-tuning below. The ratio of mass splitting is given by

$$
\frac{\Delta m_{12}^{2}}{\Delta m_{23}^{2}}=\frac{\left(1+h_{s}^{2}\right)^{2}-\left(1+h_{d}^{2}\right)^{2}}{\left(1+h_{b}^{2}\right)^{2}-\left(1+h_{s}^{2}\right)^{2}} \approx h_{s}^{2} / h_{b}^{2} \sim 0.002 .
$$

This is roughly consistent with the ratio preferred by the solar and atmospheric neutrino problems [3, 15], $\Delta m_{12}^{2} / \Delta m_{23}^{2}=0.8 \times 10^{-5} \mathrm{eV}^{2} / 0.5 \times 10^{-2} \mathrm{eV}^{2} \sim 0.0016$, where we assume the small angle MSW solution to the solar neutrino problem. The overall mass scale can be set by an appropriate choice for the $\rho$ vev, which is a free parameter in the theory.

Neutrino mixing angles. To obtain nonvanishing neutrino mixing angles, we require nonzero relative rotations in diagonalizing $M_{E}$ and $M_{L L}$. Although the matrices in Eqs. (2.12) and (2.13) are built out the same set of primordial symmetry-breaking fields, we avoid any unwanted alignments by assuming that there are generally more than one field involved in symmetry breaking at any given scale; entries of comparable size in $M_{E}$ and $M_{L L}$ are then expected to differ by order one factors. For example, consider the leading corrections, of order $h_{b}$, originating from a number of fields transforming like $\Phi_{d}^{(1)}$. While one linear combination of these fields, $\Phi_{d}^{(1)^{\prime}}$, is involved in $M_{L L}$ as we saw above, $M_{E}$ originates from the sum of two different superfield diagrams, shown in Figure 1. The second diagram involves different vertices, and thus does not depend on the same linear combination of the $\Phi_{d}^{(1)}$. Thus, it is safe to read off the order of magnitude of the neutrino mixing angles from these matrices. All terms proportional to $h_{b}$ in Eq. (2.13) (including those in the 12 and 13 entries) are diagonalized by an order one 23 rotation, $\sin ^{2} 2 \theta_{23} \sim 1$. The 12 block of the resulting matrix is then diagonalized with $\sin ^{2} 2 \theta_{12} \sim 4 h_{d}^{2} / h_{s}^{2} \sim 0.009$. The small angle MSW solution to the 
solar neutrino problem requires $\sin ^{2} \theta_{12}=3 \times 10^{-3}-1.1 \times 10^{-2}$ [15], while the range for $\sin ^{2} 2 \theta_{23}$ preferred by the atmospheric neutrino anomaly is $\sin ^{2} 2 \theta_{23}=0.4-0.6$ [2]. Thus, the model gives the desired order of magnitude relations.

Fine-tuning. We have seen that our results for neutrino mass squared splittings and mixing angles followed from the observation that the largest eigenvalues of the nontrivial matrix in Eq. (2.13) were of order $h_{b}^{2}$ and $h_{s}^{2}$, rather than both $h_{b}^{2}$. This result was a consequence of the renormalizable origin of the flavor symmetry breaking operators. While our result does not appear to involve any fine-tuning, we will now consider more precisely under what circumstances this is actually the case.

First, there was an assumption implicit in the analysis, that the 23 and 33 elements of $\Phi$ have no relative phase. If this were not the case, we would find that the order $h_{b}^{2}$ correction to $M_{L L}$ in $\Delta+\Delta^{T}$ would again have two eigenvalues of order $h_{b}^{2}$. Since the origin of $\mathrm{CP}$ violation is unclear, we could simply resign ourselves to fact that the absence of this relative phase is a restriction that we must place on the model. However, in models where CP is a good symmetry at high energies, and then spontaneously broken at some scale $\Lambda_{C P}$, it may simply be the case that $\Lambda_{C P}$ lies below the scale at which $h_{b}$ is generated. Then phases may arise elsewhere in the Yukawa textures, but will never spoil our results. The second, and somewhat more subtle point, is that the eigenvalue result follows because we have a nongeneric set of operators involving the $\Phi_{d}^{(1)}$ fields immediately below the scale where we integrate out the vector-like states. Since the Kähler potential is not protected by the supersymmetric nonrenormalization theorem, one might worry that we generate operators involving other combinations of the $\Phi_{d}^{(1)}$ fields when we run down to the scale $h_{b} M_{F}$ where these fields acquire vevs, and are themselves integrated out of the theory. There are a number of ways to address this point. First, it may be the case that $h_{b}$ is actually generated at the scale $M_{F}$ rather than below it; models exist in the literature in which $\langle\Phi\rangle$ is generated at the scale $M_{F}$, but of order $M_{F} / 16 \pi^{2}$, as a consequence of finite loop effects. The remaining Yukawa couplings may then follow from a sequential breaking of the remaining flavor symmetries at lower scales, in the way suggested earlier. Another response is to point out that there are no fields left in our model below the scale $M_{F}$ that can couple to $\Phi_{d}$ and contribute to wavefunction renormalization that may generate unwanted operators; this may persist an extensions of the theory that explains the origin of the $\Phi$ vevs, though extending the model in this way is well beyond the scope of this letter. Whether the reader chooses to view the neutrino mass relations as a prediction of the theory, as we have suggested, or take them as inputs to which undetermined coefficients are fit in a phenomenological 
approach, the flavor structure of the neutrino sector in this scenario leads to interesting predictions for the flavor structure of the squark and slepton mass matrices, as we will now discuss.

\section{The quark and lepton sector}

Like many other models with non-Abelian family symmetries, this model naturally solves the supersymmetric flavor problem. The values of the $K-\bar{K}$ mass difference and of $\epsilon^{\prime} / \epsilon$ imply that the masses of the down and strange squarks are very nearly degenerate if the quark and squark mass matrices are not aligned. In this model, the squark masses of the first two generations are degenerate in the flavor symmetric limit, and are split only by effects of the small fermion Yukawa couplings. All flavor-changing neutral current processes in the quark and charged lepton sectors are calculable in terms of the entries in Eqs. (2.6) and (2.7), which were determined in part by the requirement we obtain the neutrino phenomenology described in the previous section.

A comprehensive analysis of the flavor changing neutral current constraints on soft masses in a general supersymmetric theory has been given by Gabbiani, et al. [14. The constraints are presented in terms of the quantities $\left(\delta_{i j}\right)_{A B}$, the ratio of the $i j$ offdiagonal term in a given squark or slepton mass-squared matrix to the average diagonal entry, in the basis where the fermion mass matrices are diagonal. (Since the scalars are nearly degenerate, the ambiguity in the word "average" is irrelevant). The indices $A$ and $B$, are either $L$ or $R$, indicating the helicity of the corresponding quark or lepton. The order of magnitudes for the $\delta_{i j}$ can be determined by constructing all the $1 / M_{F}$ suppressed operators that contribute to the soft scalar masses, and to the trilinear interactions involving the Higgs fields (the A-terms). For example, we find the scalar mass squared matrices

$$
\begin{gathered}
m_{\tilde{Q}}^{2} \sim\left(\begin{array}{ccc}
m_{1}^{2}+h_{s}^{2} \lambda^{2} m^{2} & h_{c} h_{s} \lambda m^{2} & h_{s} \lambda m^{2} \\
h_{c} h_{s} \lambda m^{2} & m_{1}^{2}+h_{c}^{2} m^{2} & h_{s} m^{2} \\
h_{s} \lambda m^{2} & h_{s} m^{2} & m_{3}^{2}
\end{array}\right) \\
m_{\tilde{U}}^{2} \sim\left(\begin{array}{ccc}
m_{1}^{2}+h_{u}^{2} m^{2} & h_{u} h_{c} m^{2} & h_{u} m^{2} \\
h_{u} h_{c} m^{2} & m_{1}^{2}+h_{c}^{2} m^{2} & h_{s} m^{2} \\
h_{u} m^{2} & h_{s} m^{2} & m_{3}^{2}
\end{array}\right),
\end{gathered}
$$

where the $m$ and $m_{i}$ are generic supersymmetry-breaking masses. The mass matrix for $m_{\tilde{D}}^{2}$ is of the same form as Eq.(2.13), while $m_{\tilde{E}}^{2}$ and $m_{\tilde{L}}^{2}$ have the same form as 
$m_{\tilde{Q}}^{2}$ and $m_{\tilde{D}}^{2}$, respectively. Thus in our model, the severely constrained combination, $\left[\left(\delta_{12}^{d}\right)_{L L}\left(\delta_{12}^{d}\right)_{R R}\right]^{1 / 2} \sim\left(\lambda h_{c} h_{s} \cdot h_{d} h_{s}\right)^{1 / 2} \sim 10^{-7}$, while the bound from $K-\bar{K}$ mixing is of order $10^{-3}$ [14. We find that the remaining $\delta_{L L}$ and $\delta_{R R}$ are so much smaller than the constraints in Ref. [14], that we won't bother stating them explicitly. On the other hand, we find that the constraints from the $\left(\delta_{i j}\right)_{L R}$ are much more significant. The left-right scalar mass terms arise from the trilinear scalar interactions involving the Higgs fields. Since these interactions have the same $G_{f}^{e f f}$ symmetry structure as the Yukawa couplings themselves, the corresponding scalar mass squared matrices have the same structures as Eqs. (2.6) and (2.7), with an overall scale $A\langle H\rangle$, where $A$ has dimensions of $\mathrm{GeV}$. The most significant constraints on the model from the $\left(\delta_{i j}\right)_{L R}$ are given in Table 2, assuming $A$-parameters of $100 \mathrm{GeV}$. We translate the constraints on the $\delta_{i j}$ into lower bounds on the average quark or slepton mass. In the slepton sector, the strongest bound comes from $\mu \rightarrow e \gamma$, which forces the slepton mass to be greater than approximately $400 \mathrm{GeV}$. Since we expect all supersymmetric particle masses to be less than $\mathrm{O}(1) \mathrm{TeV}$, this would imply that an eventual improvement in the bound would rule out this model. Note however that the bound can be further relaxed if we take into account possible variation in the unknown order one coefficients that multiply the $1 / M_{F}$ suppressed operators. Such fluctuations are in fact necessary elsewhere in the model, to reproduce the Georgi-Jarlskog factors of 3 in the charged lepton Yukawa matrix. This limitation is also found in other models of flavor that cannot be embedded into a grand unified theory (e.g. Ref. [9]) ). If $\mu \rightarrow e \gamma$ is somewhat suppressed relative to our naive estimates, then the next signature of new physics would be $\tau \rightarrow \mu \gamma$, which should be seen with an order-of-magnitude improvement in experimental sensitivity. Note that potentially stronger bounds on the squark masses can be derived from $\epsilon^{\prime} / \epsilon$, but only if assumptions are made about unknown CP-violating phases. In the case in which $\mathrm{CP}$ violating phases are of order one, we find, for example, that $\operatorname{Im}\left(\delta_{12}^{d}\right)_{L R} \sim\left(A\langle H\rangle / m^{2}\right) h_{s} \lambda \sim\left[(200 \mathrm{GeV})(200 \mathrm{GeV}) /(500 \mathrm{GeV})^{2}\right] h_{s} \lambda \sim 2 \times 10^{-5}$, which is in borderline agreement with the experimental bounds for $500 \mathrm{GeV}$ gluinos.

In addition to the predictions for flavor-changing neutral currents, this model makes a striking prediction for the diagonal squark and slepton masses. Since the low-energy effective theory is constrained by the symmetry $G_{f}^{\text {eff }}$, whose non-Abelian factors are $\mathrm{U}(2)_{Q} \times \mathrm{U}(2)_{U} \times \mathrm{SO}(3)_{D}$, we expect the left-handed up and down squarks, as well as the right-handed up squarks to be nearly degenerate for the first two generations respec-

\footnotetext{
$\ddagger$ Thus, these models are consistent with a string unification of the gauge couplings, rather than a field-theoretic unification.
} 


\begin{tabular}{cccc} 
Process & Expt. Constraint & Model Prediction & Bound \\
\hline$\mu \rightarrow e \gamma$ & $\left(\delta_{21}^{\ell}\right)_{L R}<(1.4-3.8) \times 10^{-6}$ & $\left(\delta_{21}^{\ell}\right)_{L R}=\frac{\lambda m_{s} A}{m_{\tilde{\ell}}^{2}}$ & $m_{\tilde{\ell}}>(330-420) \mathrm{GeV}$ \\
$\tau \rightarrow \mu \gamma$ & $\left(\delta_{23}^{\ell}\right)_{L R}<(1.7-4.4) \times 10^{-2}$ & $\left(\delta_{23}^{\ell}\right)_{L R}=\frac{m_{b} A}{m_{\tilde{\ell}}^{2}}$ & $m_{\tilde{\ell}}>(100-130) \mathrm{GeV}$ \\
$b \rightarrow s \gamma$ & $\left(\delta_{32}^{q}\right)_{L R}<(1.3-3) \times 10^{-2}$ & $\left(\delta_{32}^{q}\right)_{L R}=\frac{m_{b} A}{m_{\tilde{q}}^{2}}$ & $m_{\tilde{q}}>(250-300) \mathrm{GeV}$
\end{tabular}

Table 2: Most significant $\left(\delta_{i j}\right)_{L R}$. The experimental constraints shown are given for $m_{\tilde{l}}=100 \mathrm{GeV}$ and $m_{\tilde{q}}=500 \mathrm{GeV}$, and scale as the square of the scalar mass. The ranges in values shown corresponds to $m_{\tilde{g}} / m_{\tilde{q}}$ and $m_{\tilde{\gamma}} / m_{\tilde{\ell}}$ varying between 0.3 and 5 . The bounds correspond to $A=100 \mathrm{GeV}$.

tively (with the third generation scalar mass differing by some order one factor), while the right-handed down squarks remain more nearly degenerate for all three generations, because of the approximate $\mathrm{SO}(3)$ symmetry. Corresponding statements can be made in the lepton sector. If we choose to think of the high energy flavor symmetry as $G_{f}$, then we can restate this result by noting that the original symmetry, which treats all three generations equally, has some remnant effect in sectors of the theory that are not directly affected by the mechanism which generates the top quark Yukawa coupling. This fact is what allowed us to obtain quasi-degenerate neutrinos, as well as the associated phenomenology described above.

\section{Conclusions}

We have presented a model with quasi-degenerate neutrinos that can account for the solar and atmospheric neutrino problems. By working with a flavor symmetry that constrains both the quark and lepton sectors, the model relates the neutrino mass squared splittings and mixing angles to quark Yukawa couplings, $\Delta m_{12}^{2} / \Delta m_{23}^{2} \sim m_{s}^{2} / m_{b}^{2}, \theta_{12} \sim$ $m_{d} / m_{s}$ and $\theta_{23} \sim 1$. These have the correct orders of magnitude to give us the small angle MSW solution to the solar neutrino problem, as well as atmospheric $\nu_{\mu}-\nu_{\tau}$ oscillations. The model naturally evades the supersymmetric flavor problem by maintaining sufficient squark and slepton degeneracy, while yielding definite predictions for the flavor structure of the superparticle mass matrices. In particular, the approximate flavor symmetry at low energies implies approximate three-generation degeneracy for the right-handed down squarks and left-handed charged leptons, respectively, while ap- 
proximate degeneracy among only the first two generations for the remaining squarks and slepton states. The model predicts $\mu \rightarrow e \gamma$ just beyond the current bound, so that we would expect this process to be detected with an order of magnitude improvement in experimental sensitivity.

\section{References}

[1] M. Dine, R. Leigh and A. Kagan, Phys. Rev. D48 (1993) 4269; Y. Nir and N. Seiberg, Phys. Lett B309 (1993) 337; See also Refs. [9] and [10], and references therein.

[2] M. Fukugita and T. Yanagida, in Physics and Astrophysics of Neutrinos, eds. M. Fukugita and A. Suzuki (Springer-Verlag, Tokyo, 1994), 1.

[3] Y. Totsuka, talk at the 18th International Symposium on Lepton-Photon Interactions, July 28-August 1, 1997, Hamburg.

[4] M. Fukugita, M. Tanimoto and T. Yanagida, hep-ph/9709388 (1997).

[5] S.T. Petcov and A. Yu. Smirnov, Phys. Lett B322 (1994) 109.

[6] D.O. Caldwell and R.N. Mohapatra, Phys. Rev. D48 (1993) 3259; Phys. Rev. D50 (1994) 3477.

[7] P. Binetruy, S. Lavignac, S. Petcov and P. Ramond, Nucl. Phys. B496 (1997) 3.

[8] H. Georgi and C. Jarlskog, Phys. Lett B86 (1979) 297.

[9] C.D. Carone, L.J. Hall, and H. Murayama, Phys. Rev. D53 (1996) 6282.

[10] C.D. Carone, L.J. Hall, and H. Murayama, Phys. Rev. D54 (1996) 2328.

[11] For a similar texture, see C.D Carone and H. Murayama, Phys. Rev. D52 (1995) 4159.

[12] L.J Hall and L. Randall, Phys. Rev. Lett. 65 (1990) 2939.

[13] C.D. Froggatt and H.B. Nielsen, Nucl. Phys. B147 (1979) 277.

[14] F. Gabbiani, E. Gabrielli, A. Masiero, and L. Silvestrini, Nucl. Phys. B477 (1996) 321. 
[15] J.N. Bahcall and P.I. Krastev, Phys. Rev. D53 (1996) 4211. 